\title{
La astronomía y su impacto en las culturas antiguas - Estudio comparativo de los casos en Egipto y el mundo Maya
}

\author{
Eduardo Rodas-Quito
}

\section{Resumen}

En las culturas antiguas, los mitos jugaron un papel muy importante para crear un sentido de orden y de identidad en las mismas, al relatar el origen del orden natural percibido y al mismo tiempo justificar el orden social y vital en el que las culturas se desenvolvían. Se estudian los mitos de la creación de dos de las grandes culturas del pasado, la maya y la egipcia, realizando un análisis de las mismas utilizando como herramienta básica la metodología planteada por Antropología Cultural, identificando en ambas algunos aspectos que dan a conocer cómo los miembros de dichas culturas concibieron el pensamiento de sus dioses al momento de la creación, desde sus perspectivas geográficas, concepciones que de algún modo, puede haberles condicionado en la forma de dar culto a los dioses, de rendirles tributo, ofrendas y/o sacrificios y en última instancia, en el destino final de sus respectivas civilizaciones.

Palabras Clave: Antropología Estructural, Mitología, Egipto, Mayas

\section{Abstract}

In ancient cultures, myths had a very important role when creating a sense of order and identity in them, by narrating the origin of the perceived natural order and at the same time, justifying the social and vital order in which cultures developed. We study the myths of the creation of two great cultures from the past, the Maya and the Egyptian, analyzing them by using as basic tool the methodology proposed by Cultural Anthropology, identifying in both of them, aspects that reveal how the members of these cultures, from their geographical perspectives, conceived the way of thinking of their gods at the moment of creation, conceptions that somehow, may have conditioned them into the way they worshiped the gods, payed them tributes, offerings and / or sacrifices and, perhaps, how their civilizations met their ultimate fate.

Keywords: Structural Anthropology, Myhology, Egypt, Mayas 
Eduardo Rodas-Quito Honduras, Tequcigalpa M.D.C., Ciudad Universitaria,email: eduardo.rodas@unah.edu.hn, Máster en Astronomía y Astrofísica, Departamento de Arqueoastronomía y Astronomía Cultural, Facultad de Ciencias Espaciales, Universidad Nacional Autónoma de Honduras (UNAH)

Fecha de Recepción: 20 de junio del 2018 / Fecha de Aprobación: 31 de octubre del 2018 


\section{INTRODUCCIÓN}

Se presentan las investigaciones realizadas sobre las culturas maya y egipcia, cuyo propósito es el de evaluar el impacto de los fenómenos astronómicos así como del entorno geográfico en el desarrollo de diversos rasgos característicos de las culturas antes mencionadas. Entre algunos de estos rasgos culturales tenemos los rituales funerarios, construcción de estructuras monumentales y creencias relacionadas con la vida espiritual posterior a la terrenal (Krupp (1994)).

El presente trabajo se enfoca principalmente en un rasgo cultural cuya importancia ha sido determinante en toda cultura: el mito, que no es más que una historia ocurrida en tiempos inmemoriales por medio de la cual las culturas tratan de explicar los diversos fenómenos de la naturaleza o el origen de tradiciones que se practicaron a lo largo de la existencia de las culturas. Se pone especial énfasis en aquellos mitos que explican las cosmogonías de los pueblos maya y egipcio. Al realizar un estudio de este tipo, se busca identificar el alcance que el conocimiento astronómico tuvo sobre la creación de estos rasgos culturales y hasta qué punto el entorno geográfico, social y ambiental influyó para que dichos rasgos evolucionaran de la forma que lo hicieron.

Para este trabajo se utiliza la metodología desarrollada por el antropólogo Claude Levy-Strauss, la que él llamo Antropología Estructural. Gracias a ella se pueden encontrar elementos o componentes que explican diversas formas de ver el mundo por parte de las culturas que son de interés del investigador. Dichos componentes de las mitologías, si se clasifican adecuadamente, dan un atisbo al pensamiento de la cultura que las generó y/o las conserva como parte de sus tradiciones y sistema de creencias (Lévi-Strauss (1985)). Otro enfoque que aporta elementos interesantes para el estudio de culturas antiguas es la utilizada por el investigador John Campbell, quien en su sistema de enseñanza de la mitología comparada, realizaba estudios en etapas, entre ellas: una inicial en la que se clasifican los mitos primitivos en varias categorías, analizando diversas historias de pueblos originarios y cazadores, según su región, y posteriormente incorporando una visión generalizadora de los temas de los mitos, en las que otras disciplinas entran en juego, como la sicología y la historia (Campbell (2013)).

Para una mejor capacidad de análisis, en el estudio se incorporan métodos desarrollados por especialistas de la historia de las religiones, uno de cuyos máximos exponentes fue el mitólogo Mircea Eliade, quien en su compilación "Metodología de la Historia de las Religiones" aporta diversos elementos que resultan muy útiles para el estudio que se presenta en este artículo (Eliade y Kitagawa (1996)).

Como ya se mencionó, con la metodología antes descrita se obtienen detalles de los mitos de las culturas maya y egipcia que permiten la identificación de rasgos en ambas culturas que permitieron la creación de tradiciones, rituales y creencias religiosas, cuyo origen esté basado en fenómenos celestes.

\section{METODOLOGÍA}

La metodología está basada en la investigación documental, en la que se busca la información pertinente en documentos tales como artículos de revistas especializadas, informes de investigación de trabajos realizados sobre las culturas de interés y libros de texto que incluyan como parte de su contenido el alcance y objetos de estudio que se buscan investigar en el presente trabajo. Con esta información se construyó un cuadro que detalla los diferentes rasgos culturales de 
las dos civilizaciones que son objeto de estudio: la maya y la egipcia. Dichos rasgos culturales se agruparán de acuerdo al fenómeno astronómico al que hagan referencia. Una vez elaborado este cuadro comparativo, se procedió a estudiar en detalle las mitologías, creencias o rituales relacionados a los mismos, con los que se espera identificar patrones que revelen mecanismos o procesos mentales que, puestos en el contexto social, geográfico y temporal de las civilizaciones que les crearon, puedan ayudar a conocer cómo la interacción entre estos elementos define aspectos o rasgos característicos de la creación y evolución de tradiciones, creencias y rituales presentes en las culturas maya y egipcia. Para el estudio documental se consultaron artículos científicos, así como libros especializados en las áreas de Astronomía Cultural, Antropología e Historia y Psicología, dado el carácter interdisciplinario de este tipo de estudios realizados en el marco de la Astronomía Cultural, en la que se vuelve necesario el concurso de varias áreas del conocimiento humano para poder comprender los diversos aspectos culturales de civilizaciones cuya visión del cosmos era integral, es decir, se conjuntaban diversos aspectos (religión, salud, mitologías, observación de fenómenos naturales) que hoy consideramos independientes entre sí. En el desarrollo de la investigación y análisis de los elementos que componen las mitologías de origen astronómico en las culturas maya y egipcia, se hace uso en primer lugar de la metodología desarrollada por el antropólogo Claude Lévi-Strauss, quien en su obra "Antropología Estructural" enumera los pasos necesarios para el análisis de mitologías (Lévi-Strauss (1985)):

a) Se aíslan dentro de cada mitología historias cortas que forman parte de la mitología bajo estudio, a las cuales llama "mitemas".

b) Se buscan relaciones entre mitemas, las cuales son generalmente opuestas una de otra, en el sentido de positivo vs. negativo, héroe vs. antihéroe, etc.

c) Se organizan estos mitemas en dos dimensiones:

i. Horizontalmente se organizan de forma sincrónica (es decir, como se van sucediendo en el tiempo).

ii. Verticalmente se organizan de forma diacrónica (no se organizan necesariamente según suceden en el tiempo, sino que se buscan "parear" mitemas cuyas historias relatadas sean de la misma naturaleza).

d) Se identifican las relaciones existentes de forma vertical y al mismo tiempo las relaciones existentes de forma horizontal en el cuadro elaborado según el inciso anterior.

Con el método antes descrito, se identifican "haces" de relaciones, como las llama Lévi-Strauss, las que constituyen las verdaderas unidades constitutivas de los mitos y en donde se rastrearán los orígenes de los mismos en fenómenos astronómicos visibles desde el sitio donde se desarrollaron las culturas bajo estudio. Una vez identificados estos haces de relaciones, se hace un análisis con ayuda de las disciplinas de la sicología, historia y geografía para una identificación más probable del origen astronómico de estos mitemas, así como del cuerpo astronómico que le originó.

\section{RESULTADOS}

Se analizaron dos mitos, uno de la cultura maya y el otro de la cultura egipcia. Ambos tienen que ver con las historias de la creación, desde el punto de vista 
particular de cada una de estas culturas. A continuación, una descripción de cómo se aplicó la metodología del inciso anterior.

i Mito de la creación maya El mito de la creación más difundido, desde el punto de vista de la cultura maya, es el que se describe en el Popol Vuh, un documento escrito en idioma maya kiché, cuyo original tiene un paradero desconocido al día de hoy. Se cree que dicho original fue elaborado por un indio maya alrededor del siglo XVI, quien habría aprendido la escritura traída por los españoles y transcribió las historias del Popol Vuh contadas por algún anciano de su pueblo. Posteriormente, este documento fue traducido y escrito en las lenguas castellana y maya kiché por Fray Francisco Ximénez, cura doctrinero en el pueblo de Santo Tomás Chichicastenango. Sin embargo, el documento original de donde lo tradujo desapareció y solo se cuenta con la versión de fray Ximénez. Posteriormente varios estudiosos han hecho sus propias versiones traducidas del documento escrito en maya por fray Ximénez, cada una adaptándolas a sus propias lenguas en términos mas ajustados a la época histórica en que realizaron dichas traducciones. La versión utilizada para el análisis propuesto corresponde a la del guatemalteco Adrián Recinos, quien la elaboró en el año 1947 (Recinos (1947)).

Se procede a descomponer el mito en sus mitemas (organizados de la forma descrita en la sección 2. (Metodología), los que se detallan a continuación:

\section{Mitema 1}

"Sólo el Creador, el Formador, Tepeu, Gucumatz, los progenitores estaban en el agua rodeados de claridad. Llegó aquí entonces la palabra, vinieron juntos Tepeu y Gucumatz, en la oscuridad, en la noche y hablaron entre sí Tepeu y Gucumatz. Hablaron pues, consultando entre sí y meditando, se pusieron de acuerdo, juntaron sus palabras y su pensamiento [...] entonces dispusieron la creación y crecimiento de los árboles y los bejucos y el nacimiento de la vida y la creación del hombre."

\section{Mitema 2}

"Entonces vinieron juntos Tepeu y Gucumatz [...] ¡Hágase así, que se llene el vacío! ¡Que esta agua se retire y desocupe el espacio, que surja la tierra y que se afirme! [...] iQue aclare, que amanezca en el cielo y en la tierra! Así dijeron. Como la niebla, como la nube y como una polvareda fue la creación, cuando surgieron del agua las montañas, y al instante crecieron las montañas [...] se realizó la formación de las montañas y los valles y al instante brotaron juntos los cipresales y pinares en la superficie."

\section{Mitema 3}

"Y dijeron los Progenitores: ¿Sólo silencio e inmovilidad habrá bajo los árboles y los bejucos? Conviene que en lo sucesivo haya quien los guarde. Así dijeron cuando meditaron y hablaron en seguida."

\section{Mitema 4}

"Al punto fueron creados los venados y las aves. En seguida les repartieron sus moradas a los venados y a las aves [...] Y estando terminada la creación de todos los cuadrúpedos y las aves, les fue dicho por el creador y formador y los Progenitores: Hablad, gritad, gorjear, llamad [...] decid, pues, vuestros nombres, alabadnos a nosotros, vuestra madre, vuestro padre; [...] ihablad, invocadnos, adoradnos!, les dijeron." 


\section{Mitema 5}

"[...] no se pudo conseguir que hablaran como los hombres, solo chillaban, cacareaban y graznaban, no se manifestó la forma en su lenguaje y cada uno gritaba de manera diferente."

\section{Mitema 6}

"Entonces se les dijo: Seréis cambiados porque no se ha conseguido que habléis. Hemos cambiado de parecer [...] Vosotros aceptad vuestro destino: vuestras carnes serán trituradas. Así será. Esta será vuestra suerte."

\section{Mitema 7}

"Probemos ahora a hacer unos seres obedientes, respetuosos, que nos sustenten y alimentes. Así dijeron. Entonces fue la creación y la formación. De tierra, de lodo hicieron la carne del hombre."

\section{Mitema 8}

"Pero vieron que no estaba bien, porque se deshacía, estaba blando, no tenía movimiento [...] Al principio hablaba, pero no tenía entendimiento. Rápidamente se humedeció dentro del agua y no se pudo sostener."'

\section{Mitema 9}

\section{"Entonces desbarataron y deshicieron su obra y creación"}

\section{Mitema 10}

"Y en seguida dijeron: ¿Cómo haremos para perfeccionar, para que salgan bien nuestros adoradores, nuestros invocadores?

\section{Mitema 11}

Y dijeron Hurakán, Tepeu y Gucumatz cuando le hablaron el Agorero, al formador, que son los adivinos: [...] Entrad, pues, en consulta, abuela, abuelo, nuestra abuela, nuestro abuelo, Ixpiyacoc, Ixmucané, haced que aclare, que amanezca, que seamos invocados, que seamos adorados, que seamos recordados por el hombre creado, por el hombre formado, por el hombre mortal, haced que así se haga. [...] y comenzando la adivinación, dijeron así: ¡Juntaos, acoplaos! ¡Hablad, que os oigamos, decid, declarad si conviene que se junte la madera y sea labrada por el Creador y el Formador, y si éste [el hombre de madera] es el que nos ha de sustentar y alimentar cuando aclare, cuando amanezca! Y al instante fueron hechos los muñecos labrados en madera. Se parecían al hombre, hablaban como el hombre y poblaron la superficie de la tierra."

\section{Mitema 12}

"Existieron y se multiplicaron; tuvieron hijas, tuvieron hijos los muñecos de palo".

\section{Mitema 13}

"Pero no tenían alma, ni entendimiento, no se acordaban de su Creador, de su Formador; caminaban sin rumbo y andaban a gatas".

\section{Mitema 14}

"En seguida fueron aniquilados, destruidos y desechos los muñecos de palo y recibieron la muerte. Una inundación fue producida por el Corazón del Cielo". 


\section{Mitema 15}

"De tzité se hizo la carne del hombre, pero cuando la mujer fue labrada por el Creador y el Formador, se hizo de espadaña la carne de la mujer."

\section{Mitema 16}

"Pero no pensaban, no hablaban con su Creador y su Formador, que los habían hecho, que los habían creado"

\section{Mitema 17}

"Y por esta razón fueron muertos, fueron anegados. Una resina abundante vino del Cielo.[...] Y esto fue para castigarlos, porque no habían pensado en su madre, ni en su padre, el Corazón del Cielo, llamado Hurakán"

\section{Mitema 18}

"Y dijeron los Progenitores, los Creadores y Formadores, que se llaman Tepeu y Gucumatz: Ha llegado el tiempo del amanecer, de que se termine la obra y que aparezcan los que nos han de sustentar y nutrir, [...] que aparezca el hombre, la humanidad sobre la superficie de la tierra. Se juntaron, llegaron y celebraron consejo en la oscuridad y en la noche; luego buscaron y discutieron y aquí reflexionaron y pensaron. De esta manera salieron a luz claramente sus decisiones y encontraron y descubrieron lo que debía entrar en la carne del hombre. Poco faltaba para que el sol la luna y las estrellas aparecieran sobre los Creadores y Formadores. A continuación entraron en pláticas acerca de la creación y la formación de nuestra primera madre y padre."

\section{Mitema 19}

"De maíz amarillo y maíz blanco se hizo su carne [...] únicamente masa de maíz entró en la carne de nuestros padres, los cuatro hombres que fueron creados [...] fueron dotados de inteligencia, vieron y al punto se extendió su vista, alcanzaron a conocer todo lo que hay sobre el mundo [...] No nacieron de mujer, ni fueron engendrados por el Creador y el Formador, por los Progenitores. Sólo por un prodigio, por obra del encantamiento fueron creados y formados [...] fueron dotados de inteligencia, vieron y al punto se extendió su vista [...] cuando miraban, al instante veían a su alrededor y contemplaban en torno a ellos la bóveda del cielo y la faz redonda de la tierra [...] grande era su sabiduría."

\section{Mitema 20}

"Entonces les preguntaron el Creador y el Formador: [...] ¡Mirad, pues! ¡Contemplad el mundo, ved si aparecen las montañas y los valles! ¡Probad, pues, a ver! Les dijeron".

\section{Mitema 21:}

"Dieron las gracias al Creador y al Formador: ¡En verdad os damos las gracias dos y tres veces! [...] Acabaron de conocerlo todo y examinaron los cuatro rincones y los cuatro puntos de la bóveda del cielo y de la faz de la tierra. Pero el Creador y el Formador no oyeron esto con gusto".

\section{Mitema 22:}

"No está bien lo que dicen nuestras criaturas, nuestras obras; todo lo saben, lo grande y lo pequeño, dijeron. [...] ¿Acaso no son por su naturaleza simples criaturas y hechuras [nuestras]? ¿Han de ser ellos también dioses? [...] Refinemos un poco sus deseos, pues no está bien lo que vemos." 


\section{Mitema 23:}

"Entonces el Corazón del Cielo les echó un vaho sobre los ojos, los cuales se empañaron como cuando se sopla sobre la luna de un espejo [...] así fue destruida su sabiduría y todos los conocimientos de los cuatro hombres, origen y principio de la raza quiché. Así fueron creados y formados nuestros abuelos, nuestros padres, por el Corazón del Cielo, el Corazón de la Tierra."

\section{Mitema 24:}

"Entonces existieron también sus esposas y fueron hechas sus mujeres. Dios mismo las hizo cuidadosamente [...] ellos engendraron a los hombres, a las tribus pequeñas y a las tribus grandes y fueron el origen de nosotros, la gente del quiché"

Una vez descompuesto el mito de la creación en mitemas, se identifican las ideas centrales de cada una de ellas, las que se pueden clasificar en:

- Ideas relacionadas con la actividad (o inactividad) de seres divinos

- Ideas relacionadas con la actividad de creaturas, las cuales pueden ser agradables o desagradables a los seres divinos

Organizándolos según estas ideas centrales, designando cada mitema con la letra $\mathrm{M}$ seguida del número correspondiente al orden en que se clasificó previamente, se obtiene la Tabla 1, que se muestra a continuación:

Tabla 1: Clasificación de los mitemas componentes del mito de creación en el Popol Vuh

\begin{tabular}{|c|c|c|c|}
\hline $\begin{array}{l}\text { Existencia y } \\
\text { meditación de } \\
\text { los dioses }\end{array}$ & $\begin{array}{ll}\text { Acciones } & \text { de } \\
\text { los dioses }\end{array}$ & $\begin{array}{l}\text { Acciones de } \\
\text { las criaturas, } \\
\text { que son } \\
\text { agradables a } \\
\text { los dioses }\end{array}$ & $\begin{array}{l}\text { Acciones de } \\
\text { las criaturas, } \\
\text { que son } \\
\text { desagradables } \\
\text { a los dioses }\end{array}$ \\
\hline M1 & M2 & & \\
\hline M3 & M4 & & M5 \\
\hline M6 & M7 & & M8 \\
\hline & M9 & & \\
\hline M10 & M11 & M12 & M13 \\
\hline & M14 & & \\
\hline & M15 & & M16 \\
\hline & M17 & & \\
\hline M18 & M19 & & \\
\hline M20 & & & M21 \\
\hline M22 & M23 & & \\
\hline & M24 & & \\
\hline
\end{tabular}

\section{ii. Mito de la creación egipcio}

Dentro de la mitología egipcia, existieron una variedad de mitos que explicaban el origen del Universo, lo que dependía de la ciudad de donde 
proviniera. El mito que se analiza es el de la ciudad conocida por los griegos como Heliópolis (o lunnu, que significa pilar en egipcio antiguo), dado que incorpora elementos que se relacionan con el campo de estudio de la arqueoastronomía. Se cree que su formulación original ocurrió en tiempos predinásticos (antes del 3,100 a.C.) siendo transmitidos por tradición oral desde esa época hasta su aparición en los Textos de las Pirámides, dentro de la pirámide del faraón de la $V$ dinastía, Wenis o Unis, (alrededor del 2,400 a.C.) en el Reino Antiguo (Belmonte (1999)). Los mitemas identificados se designan con la letra $E$, seguida del número correlativo según su aparición en el mito, de acuerdo al recuento de (Belmonte (1979)):

\section{Mitema E1:}

"No existía el cielo, no existía la Tierra, no habían sido creadas las cosas de la tierra ni las criaturas rastreras que yo levanté del $\mathrm{Nu}[\mathrm{n}]$, desde su estado de inactividad"

\section{Mitema E2:}

"No encontré un lugar donde pudiera reposar, hice un lugar en mi corazón y senté las bases en Maa y a todo le di atributos, a todo.

\section{Mietma E3:}

Yo estaba solo, porque no había escupido en la forma de Shu ni había emitido a Tefnut, ni existía nadie que trabajara conmigo."

\section{Mitema E4:}

"Senté las bases en mi corazón por mi propia voluntad y llegaron a la existencia una multitud de cosas a partir de sus nacimientos."

\section{Mitema E5:}

"Yo mismo me uní a mi mano apretada y me uní a mí mismo en un abrazo con mi sombra, sembré la semilla en mi boca por mi propia voluntad."

\section{Mitema E6:}

"Envié [aire?] en la forma de Shu, envié humedad en la forma de Tefnut"

\section{Mitema E7:}

"Y dijo mi padre Nu[n]: "Ellos debilitan a mi ojo, porque [después de mucho tiempo] de que procedieron de mí, luego de que me convertí en tres dioses a partir de uno solo [...] lloré sobre ellos y surgieron hombres y mujeres de las lágrimas que salieron de mi ojo, el cual enfureció en contra mía al saber que había puesto otro ojo en su lugar. Por tanto, le di el poder y esplendor que yo había creado. Y luego de darle su lugar en mi rostro, le hice que gobernara la tierra en toda su extensión."

\section{Mitema E8:}

"Y luego aparecí en la forma de las plantas, todos los animales rastreros, y todas las cosas que llegaron a existir."

\section{Mitema E9:}

"[Luego] di a luz a Shu y Tefnut y Geb y Nut. Geb y Nut [dieron a luz] a Osiris, Horus, Seth, Isis y Neftis a partir del vientre, uno tras otro y ellos procrean y se multiplican en esta tierra."

Analizando los mitemas en busca de las ideas centrales, se observa que todos ellos, excepto en el 7, los enunciados se expresan en primera persona e indican también la acción o inacción de quien esta narrando la historia (en este caso, 
se trata del dios $\mathrm{Ra}$ - el Sol cenital). La inacción se expresa en los mitemas 1 al 3. Su acción se expresa en los mitemas 4, 5, 6, 8 y 9. Otro aspecto que se puede identificar en este mito es que la creación se distinguen dos niveles: la creación de seres divinos y la creación de seres terrenales, comunes y corrientes. Siguiendo estos criterios, los mitemas se organizan como se presenta en la Tabla 2:

Tabla 2: Clasificación de los mitemas componentes del mito de la creación egipcio

\begin{tabular}{c|cc}
\hline Inacción por parte del dios creador Ra & \multicolumn{2}{|c}{ Acción por parte del dios creador Ra } \\
\hline E1 & E4 \\
E2 & E5 \\
E3 & \\
\cline { 2 - 3 } & Seres Divinos & Seres Terrenales \\
\cline { 2 - 3 } & E6 & E7 \\
& & E8 \\
\hline
\end{tabular}

\section{ANALISIS DE RESULTADOS}

Se analizaron los mitemas con el fin de encontrar las ideas centrales, tal como descrito en la metodología y se comenta como sigue:

Mayas: En las primeras dos columnas de la Tabla 1, se registra lo relacionado con los dioses, mientras que el siguiente par de columnas se relacionan con las criaturas que surgen por la voluntad de dichos dioses. Las ideas contrapuestas en cada caso tienen que ver con acciones o no acciones de estos seres, analizándose por tipo a continuación:

- Dioses: Se observa que éstos tienen dos estados en oposición uno respecto del otro: la primera columna se refiere a un estado reflexivo de los dioses, en el que están pasivos, sin realizar actividades de ningún tipo. En la segunda columna, por el contrario, se aprecia la acción de los dioses, que en esta mitología abarca principalmente actividades de creación (de la tierra, las plantas, animales y las diferentes clases de hombre) o de desprecio y destrucción (primero para los animales y luego para los diferentes hombres de cada creación, a excepción de los de la cuarta creación, que en lugar de ser destruidos fueron "degradados" en cuanto a sus capacidades). Es mayor el número de estados activos de los dioses que los pasivos, lo que se explica por la gran importancia de sus acciones (establecer el Cosmos y sus habitantes).

- Criaturas: Su papel se logra identificar no como de protagonistas de su propia historia, sino como seres que están a merced de la voluntad de los dioses y cuyo único propósito es el dar alabanzas a la grandeza de dichos dioses. Quienes no cumplan con este requisito, no son del agrado de los dioses y por tanto no merecen la existencia y son destruidos. 
Se observa cómo la actividad creadora se limita únicamente a la de los seres terrenales. Los dioses creadores ya existen en un mundo (¿espiritual?) y a partir de allí, por medios no descritos en el mito, ellos primero toman decisiones y luego acciones sobre crear o destruir a los seres terrenales. Es de hacer notar que estos dioses creadores, a pesar de tener estos grandes poderes de crear o destruir, no son infinitamente sabios. Esto se manifiesta por dos hechos: en primer lugar, el no haber podido crear un ser que les adorara, en la primera creación, sino que hubo que repetir varias veces este proceso, hasta que lograron crear un ser que fue demasiado perfecto, casi un dios, por lo que hubieron de rectificar y "ajustar" las capacidades de esta criatura para que no fuera a creer que también era como un dios. En segundo lugar, los dioses, a pesar de gozar de dicha cualidad de dioses, necesitaron de ayuda para su actividad creadora, como se narra en el mitema 11: "[...] Entrad, pues, en consulta, abuela, abuelo, nuestra abuela, nuestro abuelo, Ixpiyacoc, Ixmucané, haced que aclare, que amanezca, que seamos invocados, que seamos adorados, que seamos recordados por el hombre creado, por el hombre formado, por el hombre mortal, haced que así se haga". De modo que los dioses les rogaron a Ixpiyacoc e Ixmucané que les concedieran este deseo. Se identifican, por tanto, diferenciación entre los dioses, unos poderosos pero faltos de sabiduría, y otros sabios pero sin la energía o la magia necesaria para llevar a cabo la creación.

Hasta aquí, los astros no han tenido mayor relevancia en el mito maya, únicamente sirve como punto de referencia el amanecer (causado por el Sol), que en este caso vendría a ser el momento en que se debe culminar la creación, especialmente del ser que llenaría la necesidad de los dioses de ser adorados e invocados. A partir de esta relación, no es de extrañar que los mayas utilizaran los fenómenos celestes como referentes de momentos importantes de la sociedad: salidas y puestas del Sol en los solsticios y la observación del día del paso del Sol por el cenit, por citar algunos ejemplos.

Egipcios: En dos momentos de inacción (mitemas 2 y 3), Ra manifiesta una insatisfacción al no poder hallar dónde reposar ni quien le ayudara, así como un sentimiento de soledad. A causa de estos sentimientos, se puede concluir que Ra decide emprender la creación de todas las cosas, empezando con los dioses. Primero son Shu y Tefnut, a partir de la "autofecundación" de Ra. De estos dos dioses surgen los demás del panteón egipcio. A modo de resumen, en el mitema 8 se presenta a Ra (es decir, el Sol) como el padre de todas las cosas "que llegaron a existir". El mitema 7 se sale de este orden y se refiere, en primer lugar, a una tercera persona: el Nun u océano primordial, a través de quien Ra emite una especie de lamento y al mismo tiempo una justificación sobre el porqué del gobierno de una de sus partes sobre la creación: uno de sus ojos. Es este ojo el que crea a la Humanidad, cuando se dice en el mito que "Iloré sobre ellos y surgieron hombres y mujeres de las lágrimas que salieron de mi ojo, el cual enfureció en contra mía al saber que había puesto otro ojo en su lugar". De modo que la fecundidad de Ra, el dios solar por excelencia, se extiende a todas sus partes, en este caso el ojo que llora.

Por tanto, en la creación egipcia se identifican dos formas diferentes en que ésta se lleva a cabo:

- La creación de seres con consciencia (dioses y seres humanos) que ha ocurrido a partir de emisiones de fluidos de Ra: en los textos de las pirámides se dice explícitamente que Shu y Tefnut fueron escupidos por Ra (Lull (2006)), mientras que en el mitema 7 se menciona que la Humanidad ha surgido de las lágrimas de su ojo.

- Por otro lado, las demás cosas: plantas, animales rastreros y otros, no surgen de emisiones de Ra, sino que son más bien sus "encarnaciones", como 
dice el mitema 8, es decir, los seres sin una conciencia propia vendrían a ser partes o manifestaciones de este dios, por lo que la creación, fuera de los dioses y los humanos, estaría compuesta en su totalidad por elementos componentes del Sol.

\section{IV.1 Comparación entre los mitos de la creación maya y egipcia:}

Se observan dos puntos de vista muy diferentes de cómo el mundo alcanzó el orden percibido. En la sociedad maya, que se desarrolló en las selvas tropicales de Mesoamérica, los astros no tienen un protagonismo en la creación del mundo, sino que fueron dioses pre existentes los que crearon el cosmos y tienen influencia únicamente en el mundo material, no en el inmaterial (o espiritual). Si se toma en cuenta que en esta parte del mundo la mayor parte del tiempo el cielo está oculto a los ojos de sus habitantes, ya sea porque está nublado debido a su clima tropical o por la cobertura del bosque nublado en gran parte de la región, no es de extrañar que sólo se haga una distinción entre la claridad y la oscuridad, tal como se perciben el dia y la noche desde selvas tropicales y bosques húmedos. Por otro lado, en la sociedad egipcia, que se desarrolla en una región donde la mayor parte del año los cielos están claros y no hay bosques que los cubran de la vista de los habitantes de la región, el protagonista principal es el Sol, del cual surge toda la creación (tanto material como inmaterial). De sus fluidos (que se podrían asociar al rocío de la mañana y al Río Nilo mismo) surge la vida, en forma de los seres humanos (en la tierra) y de los diferentes dioses del panteón egipcio (en el mundo espiritual y quienes rigen cada parte surgida del dios Sol, manifestada como elementos naturales sin consciencia). EI Sol, en su forma de Ra, es quien comanda y dirige toda la creación, pensamiento que se deriva del hecho de vivir en el desierto, donde el Sol está siempre presente la mayor parte del año y la vida sobre la tierra gira alrededor de los ciclos percibidos en el movimiento aparente de dicho astro.

\section{Conclusión}

Del análisis realizado en la sección anterior se pueden derivar algunas conclusiones interesantes relativas a la cosmovisión y cosmogonía de las civilizaciones maya y egipcia:

1. El medio ambiente juega un papel importante para la formación y desarroIlo de las culturas. En el caso de los mayas, debido al lugar donde surge (bosque nublado tropical, selva con cobertura boscosa muy cerrada), ellos manifiestan su visión de la creación en un mundo que solo distingue la claridad de la oscuridad, sin astros que cumplan funciones claras en su cosmogonía. Por otro lado, en el caso de los egipcios, el Sol, un astro omnipresente en el ambiente del desierto, es el ser primordial de donde surge toda la vida.

2. El papel de la magia es muy diferente en cada cosmogonía estudiada. Para los mayas, se deduce de este análisis que la magia debe ser muy importante, ya que no se explica de ninguna forma cómo surgen las cosas creadas, sino que su aparición es gracias a la voluntad de los dioses involucrados. En el caso de la cultura egipcia, se da una explicación más detallada del surgimiento de lo creado, en el que atribuyen al ser creador (el Sol) y sus criaturas, fenómenos biológicos de los que surge todo lo creado. 
3. El alcance de los seres creadores es diferente en ambas culturas. Los dioses mayas solo actúan en el mundo material. Los dioses egipcios actúan tanto en el mundo espiritual como material.

4. Las personalidades y capacidades de los dioses de ambas culturas son también diferentes: los dioses mayas dudan, se equivocan y manifiestan actitudes muy humanas, el dios solar de los egipcios actúa con seguridad de lo que hace y quiere con cada una de sus acciones.

A partir de lo aquí planteado, se podrían continuar estudios que traten de relacionar estas cosmovisiones con las actitudes de los miembros de ambas culturas hacia sus dioses: ¿creerían los mayas que podían influenciar a sus dioses inseguros, mientras los egipcios no lo creyeron así? ¿Estos enfoques tendrían algún tipo de influencia sobre sus rituales y los propósitos hacia los cuales éstos estaban encaminados? Estas son preguntas interesantes que valdría la pena examinar en más profundidad en futuros estudios sobre el tema.

\section{Referencias}

Belmonte, J. (1979). Las leyes del cielo. New York: Harper Collins Publishers.

Belmonte, J. (1999). Las leyes del cielo. Madrid: Ediciones Temas de Hoy, S.A.

Campbell, J. (2013). Comparative mythology as an introduction to cross cultural studies. The Joseph Campbell Foundation.

Eliade, M., y Kitagawa, J. E. (1996). Theory of knowledge. Barcelona: Esparsa.

Krupp, E. (1994). Echoes of the ancient skies - the astronomy of lost civilizations. Estados Unidos: Dover Publications, Inc.

Lull, J. (2006). La astronomía en el antiguo egipto. Valencia, España: Universitat de València.

Lévi-Strauss, C. (1985). La estructura de los mitos (ed.), Antropología Estructural. Barcelona: Ediciones Paidós.

Recinos, A. (1947). Popol vuh: Las antiguas historias del quiché. México: Fondo de Cultura Económico. 